\title{
Comparison of African savanna elephant (Loxodonta africana) fatty acid profiles in whole blood, whole blood dried on blood spot cards, serum, and plasma
}

\author{
Jordan Wood $^{1,2}$, Larry J Minter ${ }^{2,3}$, Doug Bibus ${ }^{4}$, Michael K. Stoskopf ${ }^{2}$, Vivek Fellner ${ }^{1}$, Kimberly Ange-van \\ Heugten ${ }^{\text {Corresp. } 1,2}$ \\ ${ }^{1}$ Animal Science, North Carolina State University, Raleigh, North Carolina, United States \\ 2 Environmental Medicine Consortium and Department of Clinical Sciences, College of Veterinary Medicine, North Carolina State University, Raleigh, North \\ Carolina, United States \\ 3 North Carolina Zoo, Asheboro, North Carolina, United States \\ 4 Lipid Technologies LLC, Austin, Minnesota, United States \\ Corresponding Author: Kimberly Ange-van Heugten \\ Email address: kdange@ncsu.edu
}

Background. African elephants in managed care have presented differences in the balance between omega-3 and omega- 6 fatty acids, a situation primarily thought to be due to dietary differences between the managed animals and their free-ranging counterparts. Because of this, circulating fatty acid status is included in routine monitoring of elephant health. A method of blood collection that requires only a few drops of whole blood, dried on filter paper (DBS) and can be used for analyzing full fatty acid profiles offers advantages in clinical application.

Methods. This study compared the use of whole blood, and whole blood DBS, serum or plasma for use in evaluating circulating fatty acid composition in African savannah elephants. Samples from 6 African elephants (two males and four females) were collected during the same week at the NC Zoo, Asheboro, NC.

Results. Results found only 2 of 36 individual fatty acids and none of the 10 fatty acid groupings were different when comparing the four blood fraction sample types to each other with Mann-Whitney U-Test pairwise comparisons. Myristic acid (14:0) was lower in the DBS samples than in whole blood, serum, and plasma and pentadecaenoic acid (15:1) was slightly more concentrated in DBS and whole blood.

Discussion. Results indicate that fatty acid profile of serum, plasma, whole blood, and DBS are comparable in African elephants. The DBS method offers advantages in acquisition and handling and may be preferable to other methods in both routine health assessment of captive animals and field research on free ranging animals. 
1 Comparison of African savanna elephant (Loxodonta africana) fatty acid profiles in whole blood, whole blood dried on blood spot cards, serum, and plasma

4

5

Jordan Wood ${ }^{1,2}$, Larry J. Minter ${ }^{2,3}$, Doug Bibus ${ }^{4}$, Michael K. Stoskopf ${ }^{2}$, Vivek Fellner ${ }^{1}$, and Kimberly Ange-van Heugten ${ }^{1,2}$

${ }^{1}$ Department of Animal Science, North Carolina State University, Raleigh, NC, USA

${ }^{2}$ Environmental Medicine Consortium and Department of Clinical Sciences, College of Veterinary Medicine, North Carolina State University, 1060 William Moore Dr, Raleigh, NC 27607, USA

${ }^{3}$ North Carolina Zoo, 4401 Zoo Pkwy, Asheboro, NC, USA

${ }^{4}$ Lipid Technologies LLC, P.O. Box 216, Austin, MN, USA

Corresponding Author:

Kimberly Ange-van Heugten ${ }^{1,2}$

NC State, Department of Animal Science, 120 Broughton Drive Raleigh, NC 27695-7621 USA

Email address: kdange@ncsu.edu

\section{Abstract}

Background. African elephants in managed care have presented differences in the balance between omega- 3 and omega- 6 fatty acids, a situation primarily thought to be due to dietary differences between the managed animals and their free-ranging counterparts. Because of this, circulating fatty acid status is included in routine monitoring of elephant health. A method of blood collection that requires only a few drops of whole blood, dried on filter paper (DBS) and can be used for analyzing full fatty acid profiles offers advantages in clinical application.

Methods. This study compared the use of whole blood, and whole blood DBS, serum or plasma for use in evaluating circulating fatty acid composition in African savannah elephants. Samples from 6 African elephants (two males and four females) were collected during the same week at the NC Zoo, Asheboro, NC.

Results. Results found only 2 of 36 individual fatty acids and none of the 10 fatty acid groupings were different when comparing the four blood fraction sample types to each other with MannWhitney U-Test pairwise comparisons. Myristic acid (14:0) was lower in the DBS samples than in whole blood, serum, and plasma and pentadecaenoic acid (15:1) was slightly more concentrated in DBS and whole blood.

Discussion. Results indicate that fatty acid profile of serum, plasma, whole blood, and DBS are comparable in African elephants. The DBS method offers advantages in acquisition and handling and may be preferable to other methods in both routine health assessment of captive animals and field research on free ranging animals.

\section{Introduction}

The optimal management and conservation of African savanna elephants (Loxodonta africana) in both free-ranging and managed environments requires better understanding of their metabolic 
44

45

46

47

48

49

50

51

52

53

54

55

56

57

58

59

60

61

62

63

64

65

66

67

68

69

70

71

72

73

74

75

76

77

78

79

80

81

82

83

84

85

86

87

88

89

and nutritional status. Free fatty acids, and the triglycerides they form, are critical for the health and function of a variety of body systems and are also biomarkers for many health concerns (Nagy and Tiuca, 2017). The importance of fatty acids includes cell membrane integrity and the production of energy for the body as well as impacts on inflammatory processes, cardiovascular, liver, pancreas, and retina health (Connor, 2000, Figueiredo et al., 2017, Nagy and Tiuca, 2017). Studies primarily in rodents have shown Omega-3 fatty acids lower inflammatory responses, reduce the risk of atherosclerosis, and improve pancreatic function by reducing insulin resistance and increase reproductive success (Connor, 2000, Figueiredo et al., 2017, Fritsche, 2006, Nagy and Tiuca, 2017). While understudied in other species, correlations between dietary omega-3 and omega-6 imbalances and atherosclerosis have been noted in several species, including African elephants (McCullagh, 1972). Additionally, studies have found differences in circulating fatty acids when comparing free ranging versus managed animals for several species (Clauss et al., 2008, Clauss et al., 2007, Dass et al., 2020 \& 2021; Schmidt et al., 2009).

Most data available on African elephant free fatty acids is only available from serum or plasma samples (Clauss et al., 2003, McCullagh, 1973, Moore and Sikes, 1967, Wood et al., 2020). Plasma and serum have been considered to better reflect the short-term circulating fatty acid status of animals while whole blood is thought to provide more information on the overall fatty acid status (Baylin et al., 2005, Hodson et al., 2013, Risé et al., 2007, Thomas Brenna et al., 2018). With differing opinions on the best blood fraction to collect for fatty acid analysis and concerns about sample collection, handling, and storage, researchers have begun to look to new blood collection methods for further answers.

Alternative methods of fatty acids analysis have been successfully used to analyze human blood with a few drops of dried whole blood on filter paper cards (Armstrong, et al., 2008, Bailey-Hall, et al., 2008). These only require small volumes of whole blood to run full free fatty acid profiles and are more easily collected and stored in field research settings (Freeman et al., 2018). Because of this positive impact on field research, DBS cards have become more prevalent in wildlife research, but direct comparisons to serum, plasma, or liquid whole blood for a majority of species is lacking (Koutsos et al., 2021, Dass et al., 2020 \& 2021). This has led to the research question of how comparable DBS samples compare to more traditional samples such as liquid whole blood, serum, or plasma regarding fatty acid profiling.

The goal of this research was to: compare the results of fatty acid analysis of DBS samples to a) traditionally collected whole blood, b) serum, and c) plasma of a cohort of managed elephants maintained on a known diet.

\section{Materials \& Methods}

Animals and Diets

Six adult African elephants (2 males and 4 females) managed at the North Carolina (NC) Zoo, Asheboro, NC, USA were sampled between 8:30 and 9:30 AM within one week during July 2020. This study was approved by the NC Zoo Animal Research Committee. These animals were fed a diet of Mazuri ${ }^{\circledR}$ Hay Enhancer ${ }^{\mathrm{TM}}$, fresh cut browse, timothy hay, and daytime pasture access for grazing.

$\underline{\text { Sample Collection and Analyses }}$ 
90 Blood samples were taken under veterinary supervision via auricular veins during routine 91 monthly wellness exams. Blood was placed into 1) untreated red top vacutainer tubes, 2) lithium 92 heparin green top vacutainer tubes, 3) serum separator red and black top vacutainer tubes, and 4) plasma separator tubes with lithium heparin light green top vacutainer tubes (Becton, Dickinson and Company, Franklin Lakes, NJ, USA). All elephants were trained with positive reinforcement for regular blood collections, so restraints were not used. Previous serum samples collected and analyzed for fatty acids in 2016-2017 by Wood et al (2020) were not included in the current study and serum samples collected in this study are new and are not part of the work published in 2020. Whole blood collected in the untreated vacutainers were transferred to Perkin-Elmer Spot Saver cards (Perkin-Elmer, Waltham, MA, USA) using four spots of approximately $80 \mu \mathrm{L}$ of whole blood (approximately $320 \mu \mathrm{L}$ per card). Samples collected in serum and plasma separator tubes were centrifuged and transferred to cryovials. Cryovials of whole blood, plasma, and serum as well as dried blood spot (DBS) cards were frozen at $-80^{\circ} \mathrm{C}$ within 3 hours of collection and stored for approximately 2 months before being shipped on dry ice to Lipid Technologies (Austin, MN, USA) for a simultaneous full fatty acid profile analysis including 36 individual fatty acids and 10 fatty acid groups (Table 1). Using established methods, samples were transmethylated with acidified methanol and the fatty acid methyl esters were quantified by area percent as analyzed on routine gas chromatography (https://lipidlab.com/services/; Koutsos et al., 2021). Values are provided on a percent of total fatty acids present.

\section{$\underline{\text { Statistics }}$}

Statistical analysis was conducted to compare DBS, whole blood, serum, and plasma to each other using pairwise comparisons by the Mann-Whitney U-test with an $\alpha=0.05$ and a $U$ test statistic of 5. Pairwise comparisons included 1) DBS compared to whole blood $(U$-statistic $=5)$, 2) DBS compared to serum $(U$-statistic $=5), 3)$ DBS compared to plasma $(U$-statistic $=5), 4)$ whole blood compared to serum $(U$-statistic $=5), 5)$ whole blood compared to plasma $(U$ statistic $=5)$, and 6$)$ serum compared to plasma $(U$-statistic $=5)$. Because the sample size was small $(n=6)$ and the use of nonparametric statistics, $p$-values are not provided in this article because this method of statistical analysis leads to $p$-values that are inaccurate and do not provide valuable information. Instead of p-values, the Mann-Whitney $U$-test uses a critical value $U$-statistic for comparison, when the calculated value is less than or equal to the critical value $U$ statistic then we fail to reject the null hypothesis and there are no differences.

\section{Results}

Of the 36 individual fatty acids and 10 fatty acids groups identified, 30 individual and 10 fatty acid groups were quantifiable (Table 1). The six fatty acids that were not present in sufficient quantity to be reliably quantified within any of the blood sample types were lauric acid (12:0), 9hexadecenoic acid (16:1w5), margaric acid (17:0), heptadecenoic acid (17:1), vaccenic acid (18:1w7), and 13-octadecenoic acid (18:1w5).

Pairwise comparisons of DBS and whole blood, DBS and serum, DBS and plasma, whole blood and serum, whole blood and plasma, and serum and plasma using the Mann-Whitney U-Test, found differences between sample type only for myristic acid (14:0) and pentadecaenoic acid (15:1). Data for myristic acid (14:0) initially showed a lower concentration present in the DBS 
136 samples than in whole blood, serum, and plasma with a very large variability. This was

137 apparently due to one sample which was an obvious outlier. When statistics were run excluding

138 this outlier DBS card data for myristic acid was tighter but much lower than identified with any

139 on the other types of blood samples. The differences identified for pentadecaenoic acid (15:1)

140 concentrations were complicated by variability among the whole blood results and serum results.

141 These variations were much greater than seen for plasma and particularly for DBS sample results

142 (Table 1). No notable differences between data from frozen whole blood samples were seen

143

144

145

146

147

148

149

150

151

152

153

154

155

156

157

158

159

\section{Discussion}

Results of this study found DBS to be an acceptable method when compared to the analysis of other blood fractions examined for both individual fatty acids and fatty acid groups with the possible exception of the fatty acids, myristic and pentadecaenoic acid. This finding is consistent with studies on human blood that have found that DBS is a useful and comparable collection method for fatty acids (Armstrong et al., 2008, Bailey-Hall et al., 2008, Thomas Brenna, et al., 2018). Visual differences of interest included the lack of myristoleic acid (14:1) and pentadecylic acid (15:0) found in the DBS samples. Myristoleic acid concentrations were very low across all sample types and only a few were detectable above baseline. It is possible that differences seen between sample types was due to residue from the filter paper or external contamination, but more likely the low concentrations present were below reliable detection limits considering the expected error in sample extraction methods. The statistically significant differences for myristic acid and pentadecanoic acid (15:1) are hypothesized have been related to challenges with elution from the DBS card.

Values found in this study were similar to previous samples collected and analyzed by the authors with the same six African elephants (Wood et al., 2020). These values were also comparable to serum and plasma values collected in free-ranging elephants (Clauss et al., 2003, McCullagh, 1973, Moore and Sikes, 1967).

Results from this study were favorable for cross comparisons of important individual fatty acids and fatty acid groups including traditional essential fatty acids: $\alpha$-linolenic acid, linoleic acid, critical fatty acids: eicosapentaenoic acid (EPA) and docosahexaenoic acid (DHA), and important fatty acid groups: omega-3 fatty acids, polyunsaturated fatty acids (PUFAs), and the omega- 6 : omega-3 ratio. As mentioned in the introduction, in managed settings, it has been noted that herbivores often have higher levels of omega- 6 fatty acids in their diet and circulation leading to an inappropriate omega- $6:$ omega- 3 ratio when compared to free-ranging animals (Clauss et al., 2003, Clauss et al., 2007). This could be relevant to problems with obesity in elephants in managed care (Morfeld et al., 2016). Being able to track circulating omega-3 fatty acids, especially EPA and DHA in elephants and potentially other megavertebrates using the simpler DBS collection of a few drops of blood would greatly facilitate better monitoring across institutions.

\section{Conclusions}

Data provided in this study supports the hypothesis that fatty acid composition of whole blood, plasma, and serum are very similar in African savanna elephants. Fatty acid results from DBS 
181 samples provide a reasonably comparable approach to liquid whole blood samples, which are 182 more difficult to store and ship.

183

184

185

186

187

188

189

190

191

192

193

194

195

196

197

198

199

200

201

202

203

204

205

206

207

208

209

210

211

212

213

214

215

216

217

218

219

\section{Acknowledgements}

The authors thank the NC Zoo elephant keeper staff and veterinary staff for their assistance in collecting and processing samples during the COVID-19 pandemic.

\section{References}

Armstrong, J.M., Metherel, A.H. and Stark, K.D. (2008). "Direct microwave transesterification of fingertip prick blood samples for fatty acid determinations." Lipids 43(2): 187-196.

Bailey-Hall, E., Nelson, E.B. and Ryan, A.S. (2008). "Validation of a rapid measure of blood PUFA levels in humans." Lipids 43(2): 181-186.

Baylin, A., Kim, M.K., Donovan-Palmer, A., Siles, X., Dougherty, L., Tocco, P. and Campos, H. (2005). "Fasting whole blood as a biomarker of essential fatty acid intake in epidemiologic studies: Comparison with adipose tissue and plasma." American Journal of Epidemiology 162(4): 373-381.

Clauss, M., Dierenfeld, E.S., Bigley, K.E., Wang, Y., Ghebremeskel, K., Hatt, J-M., Flach, E.J., Behlert, O., Catell, J.C., Streich, W.J. and Bauer, J.E. (2008). "Fatty acid status in captive and free-ranging black rhinoceros (Diceros bicornis)." Journal of Animal Physiology and Animal Nutrition 92:231-241.

Clauss, M., Grum, C. and Hatt, J.M. (2007). "Fatty acid status of captive wild animals: A review." Der Zoologische Garten N.F 76: 382-401.

Clauss, M., Wang, Y., Ghebremeskel, K., Lendl, C.E. and Streich, W.J. (2003). "Plasma and erythrocyte fatty acids in captive Asian (Elephas maximus) and African (Loxodonta africana) elephants." Veterinary Record 153(2), 54-58.

Connor, W.E. (2000). "Importance of $n-3$ fatty acids in health and disease." The American Journal of Clinical Nutrition 71(1), 171S-175S. doi:10.1093/ajcn/71.1.171s

Dass, K., Koutsos, E., Minter, L.J., and Ange-van Heugten, K. (2020). “Analysis of fatty acid profiles in Eastern box (Terrapene Carolina Carolina) and common snapping (Chelydra Serpentine) turtles for wild and in-human care environments." Journal of Zoo and Wildlife Medicine. 51(3): 478-484. 
220 Dass, K., Lewbart, G.A., Muñoz-Pérez, J.P., Yépez, M.I., Loyola, A., Chen, E., and Páez-Rosas, 221 D. (2021) "Whole blood fatty acid concentrations in the San Cristóbal Galápagos tortoise 222 (Chelonoidis chathamensis)." PeerJ. 9:e11582.

223

Figueiredo, P.S., Inada, A.C., Marcelino, G., Lopes Cardozo, C.M., de Cássia Freitas, K., de 225 Cássia Avellaneda Guimarães, R., de Castro, A.P., do Nascimento, V.A., and Hiane, P.A. (2017). 226 "Fatty acids consumption: The role metabolic aspects involved in obesity and its associated 227 disorders.” Nutrients. 9(10): 1158 doi: 10.3390/nu9101158.

228

229

230

231

232

233

Freeman, J.D., Rosman, L.M., Ratcliff, J.D., Strickland, P.T., Graham, D.R. and Silbergeld, E.K. (2018). "State of the science of dried blood spots." Clinical Chemistry 64(4):656-679.

Fritsche, K. (2006). "Fatty acids as modulators of the immune response." Annual Review Nutrition 26: 45-73.

234

Hodson, L., Eyles, H.C., McLachlan, K.J., Bell, M.L., Green, T.J. and Murray Skeaff, C. (2013)

236

"Plasma and erythrocyte fatty acids reflect intakes of saturated and n-6 PUFA within similar time

238

239 frame." The Journal of Nutrition 144(1): 33-41.

240

Koutsos, E., Minter, L.J., Ange-van Heugten, K.D., Mejia-Fava, J. and Harmes, C. (2021).

241 "Blood fatty acid profiles of neritic juvenile wild green turtles (Chelonia mydas) and Kemp's

242

243 ridley turtles (Lepidochelys kempii).” Journal of Zoo and Wildlife Medicine 52(2):610-617.

McCullagh, K.G. (1973). “Are African elephants deficient in essential fatty acids?” Nature 242:

245

246 267-268.

McCullaugh, K.G. (1972). "Arteriosclerosis in the African elephant, I. Intimal atherosclerosis 247 and its possible causes." Atherosclerosis 16(3): 307-335.

Moore, J.H. and Sikes, S.K. (1967). "The serum and adrenal lipids of the African elephant." Comparative Biochemistry and Physiology 20:779-792.

251

252

Morfeld, K.A., Meehan, C.L., Hogan, J.N. and Brown, J.L. (2016). “Assessment of body

253 condition in African (Loxodonta africana) and Asian (Elephas maximus) elephants in North American zoos and management practices associated with high body condition scores." PLoS ONE 11(7): e0155146 doi: 10.1371/journal.pone.0155146.

256

Nagy, K. and Tiuca, I.D. (2017). "Importance of fatty acids in physiopathology of human body." 258 Fatty Acids. Catala, A. (ed.), IntechOpen, doi: 10.5772/67407. 
260 Risé, P., Eligini, S., Ghezzi, S., Colli, S. and Galli, C. (2007). "Fatty acid composition of plasma, 261 blood cells and whole blood: Relevance for the assessment of the fatty acid status in humans."

262 Prostaglandins, Leukotrienes and Essential Fatty Acids 76(6): 363-369.

263

264 Schmidt, D., Koutsos, E.A., Ellersieck, M.R. and Griffin, M.E. (2009). "Serum concentration 265 comparisons of amino acids, fatty acids, lipoproteins, vitamins A and E, and minerals between 266 zoo and free-ranging giraffes (Giraffa camelopardalis)." Journal of Zoo and Wildlife Medicine 267 40: 29-38.

268

269 Thomas Brenna, J., Plourde, M., Stark, K.D., Jones., P.J. and Lin, Y. (2018). "Best practices for 270 the design, laboratory analysis, and reporting of trials involving fatty acids." The American 271 Journal of Clinical Nutrition 108(2): 211-227.

272

273 Wood, J., Koutsos, E., Kendall, C.J., Minter, L.J., Tollefson, T., Ivory, E. and Ange-van

274 Heugten, K. (2020). "Circulating nutrients and hematological parameters in managed African

275 elephants (Loxodonta africana) over a 1-year period.” Zoo Biology 39(5): 345-354.

276

277 Wood, J., Minter, L.J., Stoskopf, M.K., Bibus, D., Ange, D., Tollefson, T.N., Fellner, V. and 278 Ange-van Heugten, K. (2021). "Investigation of dried blood spot cards for fatty acid analysis 279 using porcine blood." Veterinary Medicine International 2021. doi.org/10.1155/2021/6624751. 


\section{Table $\mathbf{1}$ (on next page)}

Fatty Acid (\% of Total Fatty Acids) Profile Averages and Standard Deviations (SD) of Dry Blood Spot Cards (DBS) $(n=6)$, Whole Blood $(n=6)$, Plasma $(n=6)$, and Serum $(n=6)$ Samples from Managed African Savanna Elephants (Loxodonta africana) 
Table 1. Fatty Acid (\% of Total Fatty Acids) Profile Averages and Standard Deviations (SD) of Dry Blood Spot Cards (DBS) $(n=6)$, Whole Blood $(n=6)$, Plasma $(n=6)$, and Serum $(n=6)$ Samples from Managed African Savanna Elephants (Loxodonta africana) $)^{1-6}$

\begin{tabular}{|c|c|c|c|c|}
\hline Sample Type & DBS & Whole Blood & Plasma & Serum \\
\hline Individual Fatty Acid & Average (SD) & Average (SD) & Average (SD) & Average (SD) \\
\hline Lauric acid (12:0) & ND & ND & ND & ND \\
\hline Myristic acid (14:0) ${ }^{7}$ & $0.74^{\mathrm{a}, \mathrm{x}, \boldsymbol{\pi}}(0.31)$ & $1.9^{\mathrm{y}}(0.28)$ & $2.0^{\mathrm{b}}(0.46)$ & $1.7 \div(0.34)$ \\
\hline Myristoleic acid (14:1) & $0.00(0.00)$ & $0.07(0.04)$ & $0.02(0.05)$ & $0.03(0.05)$ \\
\hline Pentadecylic acid (15:0) & $0.00(0.00)$ & $0.79(0.12)$ & $0.52(0.15)$ & $1.01(0.49)$ \\
\hline Pentadecanoic acid (15:1) & $0.61^{\mathrm{a},},(0.10)$ & $0.57^{\propto}(0.19)$ & $0.26^{\mathrm{b}, \S}(0.08)$ & $0.34 \div(0.19)$ \\
\hline Palmitic acid (16:0) & $17.8(1.98)$ & $25.4(2.83)$ & $19.2(3.04)$ & $18.9(1.67)$ \\
\hline 9-hexadecaenoic acid (16:1w5) & ND & ND & ND & ND \\
\hline Palmitoleic acid (16:1w7) & $3.5(0.85)$ & $3.8(0.92)$ & $2.9(1.22)$ & $3.1(1.12)$ \\
\hline Margaric acid (17:0) & ND & ND & ND & ND \\
\hline Heptadecaenoic acid $(17: 1)$ & ND & ND & ND & ND \\
\hline Stearic acid (18:0) & $10.1(0.94)$ & $11.8(1.92)$ & $9.5(0.84)$ & $8.9(1.31)$ \\
\hline 13-octadecaenoic acid (18:1w5) & ND & ND & ND & ND \\
\hline Vaccenic acid (18:1w7) & ND & ND & ND & ND \\
\hline Oleic acid (18:1w9) & $23.2(2.46)$ & $28.4(2.08)$ & $18.6(3.04)$ & $19.4(3.76)$ \\
\hline Linoleic acid (18:2w6) & $18.6(2.27)$ & $13.2(2.56)$ & $22.5(3.80)$ & $22.6(4.36)$ \\
\hline$\gamma$-linolenic acid (18:3w6) & $0.80(0.38)$ & $0.23(0.10)$ & $0.89(0.40)$ & $0.80(0.40)$ \\
\hline$\alpha$-linolenic acid (18:3w3) & $3.4(0.47)$ & $1.8(0.47)$ & $3.9(0.78)$ & $3.5(0.68)$ \\
\hline Stearidonic acid (18:4w3) & $0.32(0.08)$ & $0.10(0.04)$ & $0.25(0.13)$ & $0.22(0.09)$ \\
\hline Arachdic acid (20:0) & $0.34(0.14)$ & $0.32(0.08)$ & $0.14(0.02)$ & $0.11(0.05)$ \\
\hline Eicosenoic acid (20:1w9) & $0.00(0.00)$ & $0.04(0.04)$ & $0.00(0.00)$ & $0.00(0.00)$ \\
\hline Paullinic acid (20:1w7) & $0.99(0.14)$ & $0.63(0.26)$ & $0.53(0.33)$ & $0.36(0.33)$ \\
\hline Eicosenoic acid (20:2w6) & $0.29(0.11)$ & $0.25(0.10)$ & $0.35(0.11)$ & $0.36(0.11)$ \\
\hline Mead acid (20:3w9) & $0.04(0.05)$ & $0.01(0.02)$ & $0.05(0.03)$ & $0.00(0.00)$ \\
\hline h- $\gamma$-linolenic acid $(20: 3 w 6)$ & $3.6(0.48)$ & $2.2(0.50)$ & $3.6(0.83)$ & $3.7(0.75)$ \\
\hline Arachidonic acid (20:4w6) & $9.0(1.31)$ & $4.1(1.06)$ & $8.7(2.38)$ & $8.8(2.21)$ \\
\hline Eicosatrienoic acid (20:3w3) & $0.11(0.04)$ & $0.10(0.02)$ & $0.15(0.04)$ & $0.17(0.07)$ \\
\hline Eicosatetraenoic acid $(20: 4 w 3)$ & $0.57(0.35)$ & $0.39(0.17)$ & $0.93(0.37)$ & $0.97(0.34)$ \\
\hline Eicosapentaenoic acid (20:5w3) & $2.1(0.66)$ & $0.8(0.31)$ & $1.9(0.87)$ & $1.7(0.65)$ \\
\hline Behenic acid (22:0) & $0.67(0.18)$ & $0.04(0.03)$ & $0.03(0.04)$ & $0.01(0.02)$ \\
\hline Erucic acid $(22: 1 \mathrm{w} 9)$ & $0.11(0.17)$ & $0.71(0.17)$ & $0.65(0.08)$ & $0.74(0.37)$ \\
\hline Docosatetraenoic (adrenic) acid (22:4w6) & $0.35(0.10)$ & $0.38(0.20)$ & $0.35(0.07)$ & $0.38(0.06)$ \\
\hline DPA (osbond acid) (22:5w6) & $0.09(0.12)$ & $0.33(0.15)$ & $0.21(0.14)$ & $0.15(0.05)$ \\
\hline DPA (clupanodonic acid) (22:5w3) & $1.5(0.39)$ & $0.7(0.31)$ & $1.6(0.55)$ & $1.6(0.50)$ \\
\hline Lignoceric acid (24:0) & $0.30(0.08)$ & $0.09(0.08)$ & $0.04(0.04)$ & $0.03(0.02)$ \\
\hline Docosahexaenoic acid (22:6w3) & $0.36(0.13)$ & $0.72(0.37)$ & $0.24(0.10)$ & $0.22(0.08)$ \\
\hline Nervonic acid $(24: 1)$ & $0.07(0.07)$ & $0.08(0.08)$ & $0.02(0.04)$ & $0.11(0.05)$ \\
\hline
\end{tabular}




\begin{tabular}{|c|c|c|c|c|}
\hline Fatty Acid Groups & Average (SD) & Average (SD) & Average (SD) & Average (SD) \\
\hline Saturates & $30.5(1.83)$ & $40.4(1.45)$ & $31.4(3.02)$ & $30.7(2.14)$ \\
\hline Monoenes & $25.0(2.42)$ & $30.5(2.13)$ & $20.1(3.29)$ & $21.0(4.17)$ \\
\hline Poly unsaturated fatty acids (PUFA) & $41.0(3.20)$ & $25.3(3.13)$ & $45.6(6.70)$ & $45.2(6.73)$ \\
\hline Highly unsaturated fatty acids (HUFA) & $17.6(2.74)$ & $9.7(2.21)$ & $17.8(4.72)$ & $17.7(3.99)$ \\
\hline Total w3 fatty acids & $8.3(1.00)$ & $4.5(0.81)$ & $9.0(1.51)$ & $8.4(0.91)$ \\
\hline Total w6 fatty acid & $32.7(2.83)$ & $20.7(2.84)$ & $36.6(5.98)$ & $36.8(6.20)$ \\
\hline Total w9 fatty acids & $23.4(2.41)$ & $29.3(2.27)$ & $19.3(3.08)$ & $20.3(3.98)$ \\
\hline w6/w3 fatty acid ratio & $4.0(0.51)$ & $4.7(0.90)$ & $4.1(0.74)$ & $4.4(0.64)$ \\
\hline Omega 3 HUFA & $25.6(3.73)$ & $26.8(4.27)$ & $26.8(4.67)$ & $25.9(3.76)$ \\
\hline Omega 6 HUFA & $74.4(3.73)$ & $73.2(4.27)$ & $73.2(4.67)$ & $74.2(3.76)$ \\
\hline
\end{tabular}

${ }^{1}$ Fatty acids that were not in high enough concentration to be quantified included: lauric acid (12:0), 9hexadecenoic acid (16:1w5), margaric acid (17:0), heptadecenoic acid (17:1), vaccenic acid (18:1w7), and 13octadecenoic acid (18:1w5).

${ }^{2}$ Differing superscripts $(\mathrm{a}, \mathrm{b})$ in averages columns are significantly different at $(\alpha=0.05$, U-statistic $=5)$ for DBS compared to plasma

${ }^{3}$ Differing superscripts $(\mathrm{x}, \mathrm{y})$ in averages columns are significantly different at $(\alpha=0.05, \mathrm{U}$-statistic $=5)$ for DBS compared to whole blood

${ }^{4}$ Differing superscripts $(\Phi)$ in averages columns are significantly different at $(\alpha=0.05$, U-statistic $=5)$ for DBS compared to serum

${ }^{5}$ Differing superscripts $(\$, \alpha)$ in averages columns are significantly different at $(\alpha=0.05$, U-statistic $=5)$ for plasma compared to whole blood

${ }^{6}$ Differing superscripts $\left({ }^{¥, \mathfrak{f}}\right)$ in averages columns are significantly different at $(\alpha=0.05$, U-statistic $=5$ ) for serum compared to whole blood

${ }^{7}$ Outlier from DBS samples was removed for mean and SD calculations thus DBS n=5 (U-statistic $\left.=3\right)$ 